\title{
Analytics for Investigation of Disease Outbreaks (AIDO)
}

\author{
Ashlynn Daughton ${ }^{\star 1,}$, , Maneesha Chitanvis' ${ }^{1}$, Nileena Velappan ${ }^{1}$, Forest M. Altherr ${ }^{1}$, \\ Geoffrey Fairchild ${ }^{1}$, William Rosenberger ${ }^{1}$, Attelia Hollander ${ }^{1}$ and Alina Deshpande ${ }^{1}$
}

${ }^{1}$ Biosecurity and Public Health, Los Alamos National Laboratory, Los Alamos, NM, USA; ${ }^{2}$ University of Colorado, Boulder, Boulder, CO, USA

\section{Objective}

Analytics for the Investigation of Disease Outbreaks (AIDO) is a web-based tool designed to enhance a user's understanding of unfolding infectious disease events. A representative library of over 650 outbreaks across a wide selection of diseases allows similar outbreaks to be matched to the conditions entered by the user. These historic outbreaks contain detailed information on how the disease progressed as well as what measures were implemented to control its spread, allowing for a better understanding within the context of other outbreaks.

\section{Introduction}

Situational awareness, or the understanding of elemental components of an event with respect to both time and space, is critical for public health decision-makers during an infectious disease outbreak. AIDO is a web-based tool designed to contextualize incoming infectious disease information during an unfolding event for decision-making purposes.

\section{Methods}

Public health analysts of the Biology Division at Los Alamos National Laboratory curated a diverse library of historic disease outbreaks from publicly available official reports and peer reviewed literature to serve as a representation of the range of potential outbreak scenarios for a given disease. Available outbreak metadata are used to identify properties that relate to the magnitude and/or duration of the outbreak. Properties vary by disease, as they are related to diseasespecific characteristics like transmission, disease manifestation, risk factors related to disease severity, and environmental factors specific to the given location. These properties are then incorporated into a similarity algorithm ( $s$ in Figure 1 ) to identify outbreaks that are similar to user inputs.

\section{Results}

AIDO currently includes libraries for 39 diseases that are diverse across pathogen type (viral, bacterial and parasitic) as well as transmission type (vectorborne (e.g., Dengue, Malaria), foodborne (e.g., Salmonella, Campylobacteriosis), waterborne (e.g., Cholera), and person-to-person transmitted (e.g., Measles)). In addition to providing a similarity score to the user's outbreak, we provide aggregated comparisons to multiple historical outbreaks, descriptive statistics to show the distribution of property values for each disease, and extensive contextual information about each outbreak.

\section{Conclusions}

The analytics provided by AIDO allow users to interact with a unique data set of historic outbreaks and the associated metadata to contextualize incoming information and generate hypotheses about appropriate decisions. The tool is continually updated with new functionalities and additional data.

$$
\begin{gathered}
s=\frac{\sum_{i=1}^{K} w_{i} \cdot m\left(p_{i}\right)}{\sum_{i=1}^{K} w_{i}}, \\
\sum_{\text {such that } \sum_{i=1}^{K} w_{i}=1 \text { and }} 0 \leq m\left(p_{i}\right) \leq 1_{\text {. These constraints ensure that }} 0 \leq s \leq 1 .
\end{gathered}
$$

Figure 1 illustrates the algorithm used in the tool to calculate similarity scores that reflect the extent of the match between users' inputs and outbreaks in our disease libraries.

\section{Keywords}

Unfolding disease outbreak; Outbreak investigation; infectious disease; Decision-support

\section{Acknowledgments}

This project is supported by the Chemical and Biological Technologies Directorate Joint Science and Technology Office (JSTO), Defense Threat Reduction Agency (DTRA), and the National Biosurveillance Integration Center (NBIC) of the Department of Homeland Security (DHS).

\section{*Ashlynn Daughton}

E-mail: adaughton@lanl.gov 